\title{
Estrategias metodológicas, aprendizaje colaborativo y TIC: un caso en la Escuela Complutense Latinoamericana
}

\section{Methodological strategies, collaborative learning and ICT: a case in Latin American Complutense School}

\author{
Jose Manuel SÁEZ LOPEZ y Jose María RUIZ RUIZ \\ Universidad Complutense de Madrid y Universidad de Castilla la Mancha
}

Recibido: Octubre 2011

Aceptado: Enero 2012

\section{Resumen}

El presente estudio analiza la práctica pedagógica desarrollada desde diferentes estrategias metodológicas y la integración de las TIC en el contexto de la Escuela Complutense Latinoamericana en Puebla, Méjico. La investigación se desarrolla con una complementariedad metodológica y una triangulación de métodos. Se concluye que las estrategias de Aprendizaje Basado en Problemas y el Aprendizaje Colaborativo vinculados a una integración reformista de las tecnologías, aportan beneficios notables en la práctica en el aula. En este sentido, se desarrolla la autonomía, la resolución de problemas, toma de decisiones y habilidades de pensamiento crítico.

Palabras clave: Aprendizaje Activo; Aprendizaje Basado en Problemas (ABP); Formación Docente; Pizarra Digital Interactiva (PDI); Tecnologías de la Información y la Comunicación (TIC).

\begin{abstract}
This study examines the pedagogical practice developed from different methodological strategies and ICT integration in the context of Latin American Complutense School in Puebla, Mexico. The research develops a methodological complementarity and triangulation methods. We conclude that the strategies of problem-based learning and collaborative learning linked to reformist integration of technologies provide significant benefits in classroom practice. In this sense, autonomy, problem solving, decision making and critical thinking skills are developed Keywords: Active Learning; Information and Communication Technologies (ICT); Interactive Whiteboard (IWB); Problem-Based Learning (PBL), Teacher Training.
\end{abstract}

El presente estudio se desarrolla en el contexto de la Escuela Complutense Latinoamericana, impulsado por la Fundación Complutense, en la Universidad de la Benemérita Universidad Autónoma de Puebla BUAP en Puebla, Méjico. El título del curso es Estrategias Metodológicas prácticas para la Evaluación de las Competencias 
Profesionales, que se desarrolla en dos semanas con 5 horas diarias con un grupo de 44 alumnos de 4 países distintos, Méjico, Venezuela, Colombia y Ecuador.

Los alumnos cuentan con distintos perfiles profesionales, como gestión y administración pública, derecho, directores y docencia universitaria. La mayoría de los participantes están vinculados a la docencia universitaria con una mayoría de docentes en el área artística

El curso presenta una oportunidad para aplicar una serie de estrategias metodológicas aprovechando las ventajas de la Tecnología Educativa, en este sentido, se aplica el Aprendizaje Basado en Problemas (ABP) o Problem Based Learning, y se aplica asimismo la estrategia de Aprendizaje Colaborativo con la técnica específica de puzle o Jigsaw, utilizando las Tecnologías de la Información y la Comunicación desde una perspectiva o paradigma reformista (Aviram, 2002) que tiene en cuenta la perspectiva metodológica aplicada con las Tecnologías.

La perspectiva metodológica que se aplica en el grupo objeto de estudio guarda una coherencia con el desarrollo y adquisición competencias, en este sentido, la práctica educativa está orientada a una actividad por parte del estudiante a través de actividades grupales en las que existe una interacción y enriquecimiento. La metodología orientada a resolver problemas de forma autónoma y responsable, tratando de desarrollar unos conocimientos, habilidades y actitudes en el estudiante para que sea capaz de desenvolverse en cualquier situación de un modo autónomo, eficaz y responsable.

A partir de la práctica pedagógica desarrollada, se desarrolla una complementariedad metodológica con una triangulación de métodos, pues se analizan las actividades desarrolladas desde una perspectiva cualitativa, que nos aporta información relevante en el estudio. Por otra parte, se aplica un cuestionario, con un enfoque cuantitativo que aporta datos descriptivos.

\section{Marco teórico}

En un contexto vinculado a la educación superior se propiciando un diseño y desarrollo del currículo a partir de un aprendizaje en la acción cuando se aplica un aprendizaje por competencias.

Por ello, el cambio está fundamentalmente en el "cómo" se pueden aprender los contenidos (en un contexto donde la información es fácilmente accesible) y afecta a la planificación, a la metodología y a la evaluación. Se requieren nuevos marcos de trabajo, colegiados, donde tenga cabida el desarrollo de experiencias de aprendizaje atractivas e integradas. (Cano, 2008)

Calvo et al (2010) proponen que en una evaluación continua basada en competencias, en la que podemos disponer tanto de una evaluación puramente sumativa caracterizada por asignar calificaciones numéricas, así como por definir siempre los objetivos a alcanzar, como, dando un paso más en el proceso de enseñanza, de una formativa que busque mejorar progresivamente el aprendizaje del alumno, informando pero no puntuando, sirviendo a su motivación y proporcionando al profesor la información necesaria para mejorar y reorientar el proceso de aprendizaje, al tiempo que ofrece una visión 
de las dificultades y de los progresos que los estudiantes van realizando a lo largo del curso, tratando de crear en ellos unos hábitos de trabajo, así como un sentido de la responsabilidad y capacidad de autoaprendizaje.

La planificación de la docencia con el enfoque de diseño curricular; la comprensión de los procesos de aprendizaje y el adecuado tratamiento de los elementos más influyentes; las metodologías de problemas, casos y proyectos; la evaluación como componente formativo del currículo y las técnicas de evaluación auténtica; etc. forman parte de la mayoría de los programas de formación para el profesorado universitario. (Yañiz, 2008)

Para una correcta aplicación y coherencia con un proceso de enseñanza y aprendizaje por competencias, es necesario un enfoque metodológico que aplique estrategias y técnicas metodlógicas activas que propician un protagonismo del alumno en su proceso de aprendizaje, coherentemente con un paradigma mediacional centrado en el alumno.

Ante los cambios de la sociedad actual se requiere flexibilidad y un nuevo enfoque a la hora de enseñar y aprender, pues dividir los conocimientos en diferentes asignaturas no plantea una respuesta ante la necesidad de un conocimiento integrado, derivado de la propia definición de competencias que incluye conocimientos, destrezas, habilidades y actitudes para llevar a cabo tareas diversas de forma adecuada.

Cano (2008) considera que la atención se focaliza en el aprendizaje más que en la enseñanza con un rol activo por parte del alumno que debe:

- Revisar el modo en que entendemos nuestra función docente.

- Pasar de transmisores de contenidos a facilitadores de oportunidades de crecimiento.

- Pensar tanto en el "qué" queremos que aprendan los alumnos como en el "cómo" creemos que pueden aprenderlo (y, por supuesto, en el "para qué").

- Reducir sesiones presenciales dedicadas al conocimiento conceptual sobre el que tradicionalmente pivotaban muchas asignaturas para buscar vías de apropiación del mismo a partir de trabajo autónomo (permitiendo así que las sesiones presenciales se dediquen al aprendizaje cooperativo, al debate, a la construcción de conocimiento).

El hecho de aprender por competencias lleva implícito un cambio en el proceso de enseñanza aprendizaje, para tratar de impulsar un aprendizaje activo por parte del alumno, orientado a desarrollar habilidades, destrezas, actitudes y conocimientos que le permita desenvolverse en cualquier situación. Al propiciar un cambio en el papel activo del alumno, cambia el rol del docente que pasa de transmisor de contenidos a mediador aprendizajes. Se plantean como estrategias y técnicas adecuadas aquellas que hacen referencia al aprendizaje basado en problemas (ABP), aprendizaje colaborativo, la técnica puzle y el uso de las TIC. El instrumento de evaluación que mejor se adecua a los citados enfoques es el portafolio, que además puede enfocarse como portafolio digital.

En este sentido, los procesos educativos deben responder a las demandas sociedad de la información y del conocimiento, que presentan cambios vertiginosos que influyen 
en nuestras condiciones de vida, en contextos académico y profesionales. Nuestra responsabilidad como docentes es formar a los ciudadanos del siglo XXI, para que puedan desarrollarse como individuos, posibilitando un acceso a la información y transformar esta gran cantidad de información en conocimiento, lo que no es una tarea fácil, pues se necesitan unas habilidades de pensamiento crítico.

En el estudio SITES2006, analizado en el libro de Law et al (2008) aporta información respecto a que el acceso al ordenador es una condición necesaria pero no suficiente para el uso de las TIC en el aprendizaje y en la enseñanza, en definitiva, es necesario un cambio en el modo de plantear la metodología didáctica, utilizando enfoques activos, participativos y colaborativos que propician una actividad y protagonismo al alumno por encima de enfoques tradicionales centrados en la instrucción directa y masiva por parte del profesor.

Aseguran, por tanto, que el hecho de aumentar el nivel de acceso a los ordenadores, en sí mismo no conduce a más experiencias de aprendizaje que lleven a resultados con las TIC para los estudiantes. En este estudio se asegura, asimismo, que la adopción de las TIC de por sí no determina la orientación pedagógica, como lo demuestra la observación de que en algunos sistemas que utilizan prácticas TIC exhibieron una mayor orientación profesional.

El hecho de contar con tecnologías en el aula es beneficioso pero claramente insuficiente, pues se deben tener en cuenta los aspectos pedagógicos relativos al proceso de enseñanza aprendizaje. Area (2007) afirma que no sólo es importante emplear didácticamente ordenadores y demás artilugios digitales para las tareas docentes y de aprendizaje, sino que el tipo de prácticas debieran responder a ciertos principios y criterios de calidad pedagógica.

\section{Aprendizaje colaborativo}

En la sociedad de la Información se dan unas exigencias para trabajar de forma colaborativa tratando de beneficiar al grupo compartiendo aportaciones y conocimiento, en contextos académicos y laborales. Como docentes, tenemos el reto y la responsabilidad de enseñar a los alumnos a trabajar de forma colaborativa para propiciar una preparación que posibilite el desarrollo de profesionales competentes.

Scagnoli (2005) aprecia en su práctica investigadora unos comentarios en las evaluaciones finales de los cursos en los que trabaja. En su práctica valora que existe una satisfacción por parte de los estudiantes como de los docentes con este tipo de actividades.

Se trata de aprender conocimientos y habilidades para una aplicación eficiente, saber comunicarse, compartir y discutir decisiones. Se potencia un espíritu emprendedor y una autonomía, tomando decisiones y valorando los distintos puntos de vista y perspectivas de otros compañeros. El aprendizaje colaborativo posibilita interacciones entre individuos, en las que los alumnos se comunican, expresan y desarrollan un pensamiento crítico.

Los beneficios del aprendizaje colaborativo se citan por teóricos importantes en este campo, como Johnson and Johnson (1986) y Waggoner (1992), resaltando: Interdepen- 
dencia positiva, promoción a la interacción, responsabilidad individual, desarrollos de habilidades en el trabajo en comunidad, e interacción positiva. Sheridan et al (1989) subrayan que este tipo de aprendizaje prepara a los alumnos como investigadores, a través de una satisfacción y motivación.

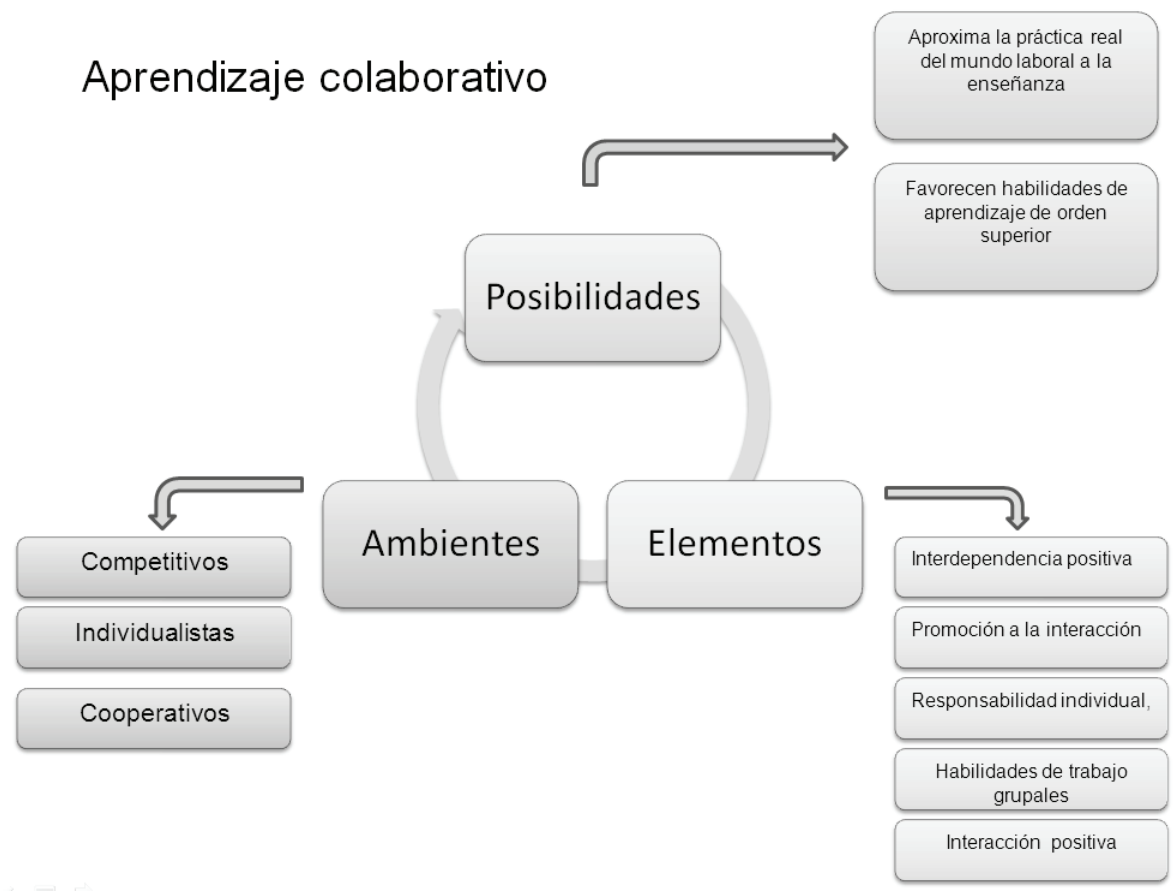

Figura 1: Elementos del aprendizaje colaborativo. Sáez López, J.M (2011)

Barab, et al (2001) aseguran que éste tipo de aprendizaje de lugar a una coconstrucción de significado al compartir experiencias personales. Aseguran que los entornos virtuales mejoran las oportunidades de comunicación e investigación con modelos educativos participativos. 


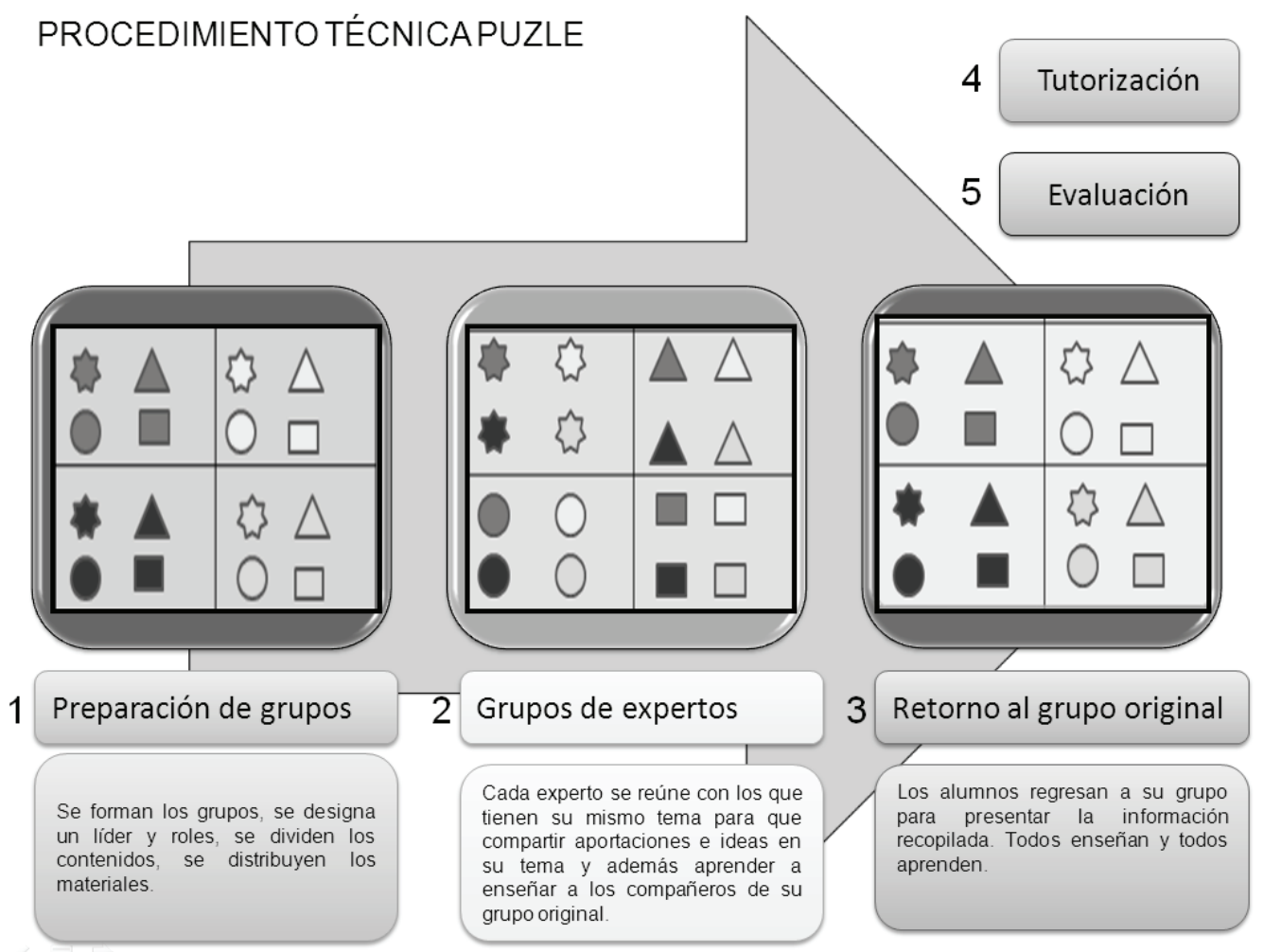

Figura 2: Aprendizaje Colaborativo. Técnica Puzle o Jigsaw. Sáez López, J. M. (2011)

Brufee, (1987) concluye en una serie de investigaciones en educación primaria y secundaria, que los alumnos aprenden menos en contextos en que se fomenta una competencia o individualidad, y aprenden mejor en contextos o situaciones de colaboración. El aprendizaje colaborativo implica superar modelos tradicionales centrados en el profesor, aportando un protagonismo activo al alumnado, el profesor pasa a facilitador. Se adquieren habilidades de orden superior coherentes con el aprendizaje por competencias, es decir, pensamiento crítico, metacognición, resolución de problemas y toma de decisiones. Otras ventajas son la motivación, mayor autonomía del estudiante y enfoques vinculados al constructivismo

\section{Aprendizaje Basado en Problemas}

Se define Aprendizaje Basado en Problemas (ABP) como un enfoque pedagógico que transforma el proceso enseñanza-aprendizaje superando enfoques tradicionales y aportando un protagonismo por parte de los estudiantes, pues éstos desarrollan sus habilidades en situaciones y problemas vinculados a la vida real. Barrows (1986) defi- 
ne $\mathrm{ABP}$ como método de aprendizaje basado en el principio de usar problemas como punto de partida en la adquisición e integración de los nuevos conocimientos.

Esta estrategia es coherente con un aprendizaje por competencias, pues las competencias cognitivas y de alto nivel de cognición implican una toma de decisiones, autonomía, habilidades de pensamiento crítico y resolución de problemas.

Al plantear el problema debe ser interesante, práctico y debe estar vinculado a la práctica real, incluso puede ser un problema real resuelto en su día o todavía pendiente de resolver en un contexto real. Cuando se plantea el problema se puede enfocar y redactar un supuesto con detalles centrados a una solución o plantear un problema más abierto con pocos datos en los que se pueden plantear soluciones muy divergentes.

Rue et al (2009) aportan un estudio con 1090 estudiantes de distintas universidades, en las que se observó que los estudiantes muestran mayores preferencias por las actividades de aprendizaje que vinculan los conocimientos teóricos con la práctica o sus aplicaciones específicas, como la resolución de problemas o el desarrollo de proyectos.

La resolución del problema no es el objetivo prioritario del aprendizaje basado en problemas, aunque pueda incluirse entre los que persigue este estrategia de aprendizaje. A nuestro entender, lo más importante a destacar son dos aspectos: La movilización, por parte de los estudiantes de recursos procedentes de diversas fuentes y el hecho de que, mediante el ABP, aprenden a aprender bien a partir de los retos mismos planteados por el problema, bien con la guía o el apoyo del tutor que actúa como facilitador. (Rué et al, 2011,26)

Gijbels et al (2003) tratan de responder en un estudio a los efectos del ABP en el conocimiento y las habilidades, y qué factores o moderadores actúan en los efectos del ABP. Los resultados indicaron que los métodos de evaluación que se centran más en el reconocimiento mostraron efectos significativamente negativos significativos para casi todo el conocimiento y favorecen el enfoque del aprendizaje tradicional. Métodos de evaluación que se centraron más en la aplicación del conocimiento mostraron mayores y mejores efectos en los entornos de aprendizaje ABP frente a los tradicionales. Los investigadores declararon que cuánto mejor evalúe el instrumento las habilidades de los estudiantes, con mayor claridad se aprecian los efectos del ABP. 


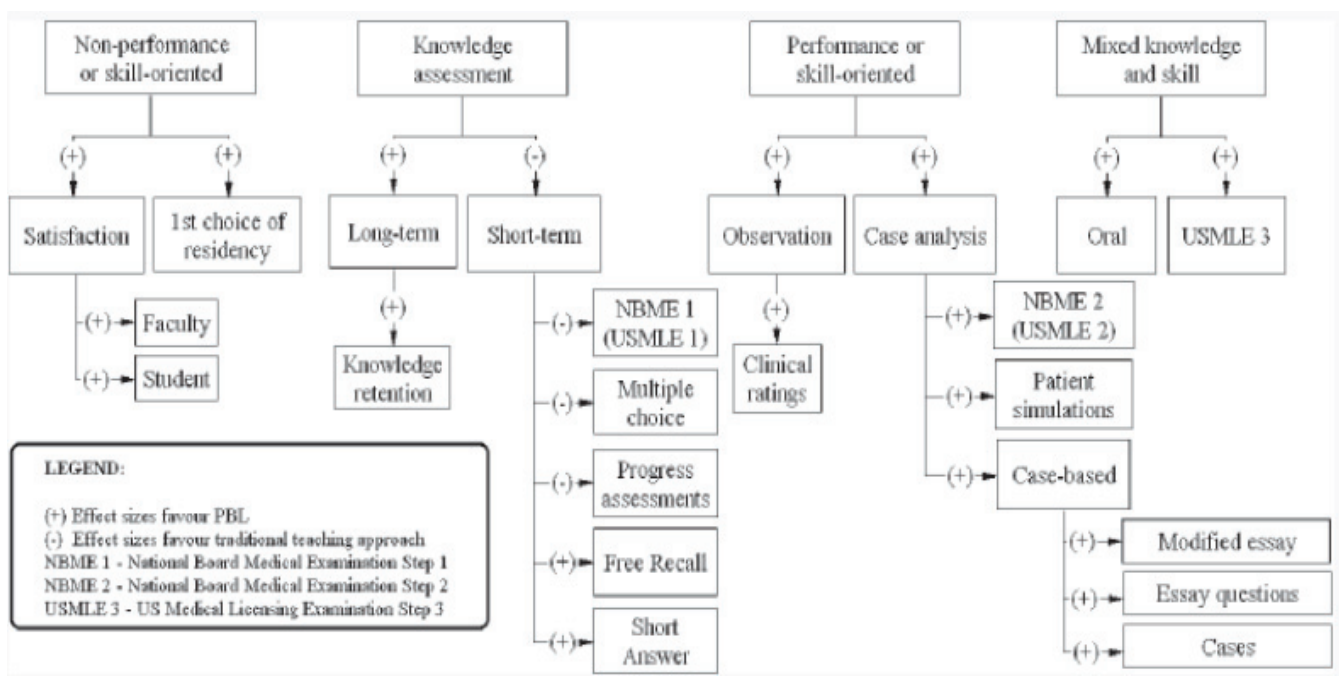

Figura 3: Mapa de resultados de aprendizaje. Gijbels et al (2003). Fuente: Strobel, et al (2008)

\section{Modelos aplicables con las Tecnología Educativa (Ed. tech)}

Desde la perspectiva del ABP y el aprendizaje colaborativo como enfoques activos en el que el alumno es protagonista de su proceso de aprendizaje, las TIC son herramientas que aportan diversas opciones interesantes que se tienen en cuenta. Se proponen y plantean varios modelos:

\section{- Exposición- solución del problema a través de PDI o Póster}

El alumno puede proponer la solución encontrada al problema, y sus aportaciones a través de una exposición oral en la Pizarra Digital o se puede plantear el diseño de un póster que sintetiza la solución al problema planteado. Las herramientas para su diseño son Power Point o Glogster en caso de un enfoque dinámico multimedia. Por supuesto se trata de proyectar en contenido en un proyector o PDI. Dulac (2006) indaga en las valoraciones de docentes y discentes relativas al uso de la PDI, y concluye que la pizarra digital es una herramienta aceptada por la generalidad del profesorado por 3 razones: porque es de fácil uso, mejora rápidamente la enseñanza y el aprendizaje y potencia la creatividad. Estas características son esenciales en la técnica citada. 


\section{- Puzle usando videoconferencia}

El uso de herramientas como Skype, Vokle, Anymeeting y otras aplicaciones enfocadas a Webinnars pueden ser utilizados alternativamente para que los sujetos de los grupos mantengan un intercambio de información tan importante en este tipo de actividades en sus diferentes fases.

\section{- Wikis}

Las wikis son páginas web que pueden ser editadas por los usuarios añadiendo contenido de todo tipo. Es un recurso interesante por la riqueza y la diversidad de fuentes. No obstante a veces se critica que carecen de precisión científica y fiabilidad. Como recurso en el enfoque colaborativo es realmente interesante.

\section{- Uso de presentaciones con Power Point o Prezi}

El alumno puede apoyarse en las tradicionales diapositivas utilizando el "Power Point" o programas similares, o incluso utilizar la aplicación Web Prezzi que aporta un entorno más dinámico, posibilitando incluso presentaciones online entre varios docentes.

\section{- Entornos Virtuales de Aprendizaje (E.V.A)}

Los trabajos aportados se añaden en el entorno o Campo Virtual, WebCT de acceso público a todos los alumnos. El profesor puede subir el problema a la plataforma y los alumnos pueden tratar de resolverlo a través de esta herramienta.

\section{- Intercambio de información con herramientas síncronas y asíncronas}

En caso de que la resolución del problema sea grupal, se necesita un intercambio de información e interacción constantes, por lo que se puede ser útil contar con herramientas asíncronas como el correo electrónico, y síncronas, como la videoconferencia o chat.

\section{- Posibilidad de usar Blogs o diseño Web para un portafolio digital}

Los alumnos pueden describir sus actividades, plasmar el proceso en el portafolio y subirlo a un Blog con la herramienta web gratuita Blogger o Wordpress. Para una evaluación se pueden combinar distintas técnicas, como exámenes orales, escritos, test o casos prácticos, sin embargo el portafolio digital mantiene una coherencia mayor con los enfoques citados. La Utilización del portafolio como recurso de evaluación da al alumnado y al profesorado una oportunidad para reflexionar sobre el proceso de aprendizaje de los estudiantes. 


\section{Marco metodológico}

La presente investigación parte de una complementariedad metodológica con una triangulación de métodos, pues se analizan las actividades desarrolladas desde una perspectiva cualitativa y cuantitativa. Se analiza la práctica pedagógica desarrollada y las opiniones y actitudes de los docentes respecto a las estrategias metodológicas aplicadas y la integración de las TIC.

El planteamiento del problema que se formula es: ¿Es posible aplicar unos enfoques metodológicos activos, vinculados al aprendizaje por competencias integrando las Tecnologías? ¿Se mantiene una coherencia y eficacia en los aprendizajes y en el desarrollo de conocimientos, habilidades y actitudes a través de prácticas activas con el uso de la Tecnología educativa?

Como objetivos en la investigación, se formulan:

- Analizar la práctica en el aula, con una aplicación de las estrategias metodológicas ABP y aprendizaje colaborativo.

- Obtener información de los participantes respecto a la idoneidad y eficacia de las estrategias metodológicas desarrolladas.

- Valorar el uso de la Tecnología Educativa, a través de Video conferencias, material multimedia, software de accesibilidad y Blogging

- Indagar en la práctica pedagógica en el aula a través en los distintos grupos heterogéneos, de distintos países, y en las conclusiones que formulan en su autoevaluación.

El contexto en el que se desarrolla el estudio es la Escuela Complutense Latinoamericana en Puebla, Méjico, el título del curso es Estrategias Metodológicas prácticas para la Evaluación de las Competencias Profesionales.

La muestra es de 44 profesionales, con un 68,2 \% de mujeres y un 31,8 \% de hombres. Se dan diversas disciplinas, un $93,2 \%$ son profesores en la docencia universitaria el $6,8 \%$ son puestos de gestión y administración pública y otras disciplinas. Por otra parte, el 84,1\% son de Méjico, el 2,3 \% de Venezuela, el 9,1\% de Colombia y el 4,5\% de Ecuador. En definitiva, la selección de la muestra es no probabilística e intencional (los sujetos que se inscriben en el curso). No existe mortalidad experimental.

La práctica llevada a cabo en el curso desarrollado en Puebla (Méjico) implica la aplicación de una serie de estrategias metodológicas:

La primera actividad es la aplicación del ABP usando las TIC y un enfoque Intercultural. Se trata de analizar y comparar el sistema educativo español y el sistema educativo mejicano. El problema que se plantea tiene una perspectiva hacia la Educación comparada, analizando el sistema educativo español y mejicano desde los datos oficiales, leyes, informes y documentos, así como analizar la situación de los dos sistemas desde la aportación de profesionales que aportan información cualitativa. Se contacta con España con dos maestros por Skype para entrevistarles. 
Otra actividad implica utilización de herramientas de accesibilidad para discapacitados utilizando la estrategia ABP. Nuestro problema es integrar a alumnos discapacitados en el aula. Se plantea una situación en la que se deben atender a unos alumnos con necesidades aplicando las herramientas de accesibilidad JAWS, zoom (varias opciones), dispositivos braille, periféricos, impresoras braille, head mouse, etc. Se analiza la información, pues contamos con una clase de 45 alumnos en un entorno universitario, 2 de ellos tienen ceguera total, 3 más son ambliopes, otro tiene sordera total y otro es hipoacúsico. Por último, otro alumno no puede mover los brazos.Se plantean como problemas: ¿Cómo organizas la clase? ¿Salen fuera del aula? ¿Qué herramientas utilizas? ¿Cómo se organizan los grupos? Se debe aportar una solución en la que se necesitan recursos económicos y otra solución en la que no se disponen de recursos económicos.

Otra actividad que aporta una perspectiva de Aprendizaje Colaborativo se refiere al diseño de un blog para educación medioambiental utilizando las TIC. En este caso se trabaja con la estrategia de aprendizaje colaborativo, con la técnica puzle o "Jigsaw". Se trata de desarrollar un blog en el que se reflejan las conclusiones y aportaciones de los grupos. El enfoque es una discusión respecto a la teoría del calentamiento global. Por falta de espacio no se detallan otras actividades.

A partir de la experiencia previa y el trabajo en el curso se analizan las opiniones, actitudes y valoraciones de los sujetos de la investigación, Se aplica un cuestionario CP1 online con Google Docs integrado en el blog del investigador en el que se también se pueden verificar los participantes del curso y sus agrupaciones http://ticjm.blogspot.com . El cuestionario tiene un enfoque cuantitativo que aporta datos descriptivos relativos al uso de las estrategias metodológicas aplicadas en el curso. La fiabilidad del cuestionario se mide por alfa de cronbach cuyo valor es de 0,64. El nivel de significación es de 0,05.

Los resultados que se obtienen a partir del cuestionario muestran valores muy positivos respecto a la aplicación de la estrategia $\mathrm{ABP}$, con valores positivos de los sujetos de la muestra (ítem 1). Se aprecian valores similares respecto a la técnica puzle o "jigsaw" dentro de la estrategia de aprendizaje colaborativo, con una amplia aceptación por parte de los docentes (ítem 2). 


\begin{tabular}{|c|c|c|c|c|}
\hline \multirow{2}{*}{$\begin{array}{l}\text { Valoración de Estrategias Metodológicas aplicadas en } \\
\text { el grupo ECL }\end{array}$} & \multicolumn{2}{|c|}{ - } & \multicolumn{2}{|c|}{+} \\
\hline & 1 & 2 & 3 & 4 \\
\hline $\begin{array}{l}\text { 1.-La estrategia de ABP (Aprendizaje Basado en Problemas) es eficaz } \\
\text { y efectiva en el proceso de enseñanza aprendizaje }\end{array}$ & 0 & 0 & 25 & 75 \\
\hline $\begin{array}{l}\text { 2.- La técnica Puzle aporta un modo dinámico de enfocar el aprendiza- } \\
\text { je }\end{array}$ & 0 & 0 & 61,4 & 38,6 \\
\hline $\begin{array}{l}\text { 3.- La lección magistral debe mantenerse como eje principal en el } \\
\text { proceso metodológico }\end{array}$ & 0 & 40,9 & 52,3 & 6,8 \\
\hline $\begin{array}{l}\text { 4.- El manejo de las TIC aporta unas herramientas esenciales para } \\
\text { trabajar por competencias }\end{array}$ & 0 & 0 & 25 & 75 \\
\hline $\begin{array}{l}\text { 5.- Para trabajar por competencias es esencial un enfoque activo cen- } \\
\text { trado en el alumno }\end{array}$ & 0 & 0 & 4,5 & 95,5 \\
\hline $\begin{array}{l}\text { 6.- Las competencias pretenden crear ciudadanos eficientes y competi- } \\
\text { tivos dejando en un segundo plano el desarrollo de los valores }\end{array}$ & 22,7 & 36,4 & 27,3 & 13,6 \\
\hline
\end{tabular}

Tabla 1: Valoración de Estrategias Metodológicas aplicadas en el grupo ECL

En cuanto al hecho de que las TIC aportan unas herramientas esenciales para trabajar por competencias, los valores aportados son muy positivos, con el respaldo de toda la muestra (ítem 4). En lo que respecta al indicador que valora que trabajar por competencias es esencial un enfoque activo centrado en el alumno obtiene los resultados más altos del cuestionario, con un 86,6 \% que aporta el valor más alto (ítem 5).

Por otra parte, existe cierta división respecto a si la lección magistral debe mantenerse como eje principal en el proceso metodológico, pues el 59,1\% de la muestra aporta valores positivos respecto esta afirmación, mientras que un 40,9\% cree que la lección magistral no debe mantenerse como eje principal desde el punto de vista metodológico.

Por último, se plantea si las competencias pretenden crear ciudadanos eficientes y competitivos dejando en un segundo plano el desarrollo de los valores (ítem 6). En esta afirmación existe una división de opiniones, con un 59,1\% opina que esta afirmación no es cierta, pues las competencias desarrollan valores por definición, mientras que un $40,9 \%$ está de acuerdo con la afirmación del ítem que asegura que las competencias tienen un enfoque orientado a la eficiencia dejando en un segundo plano la formación en valores. 


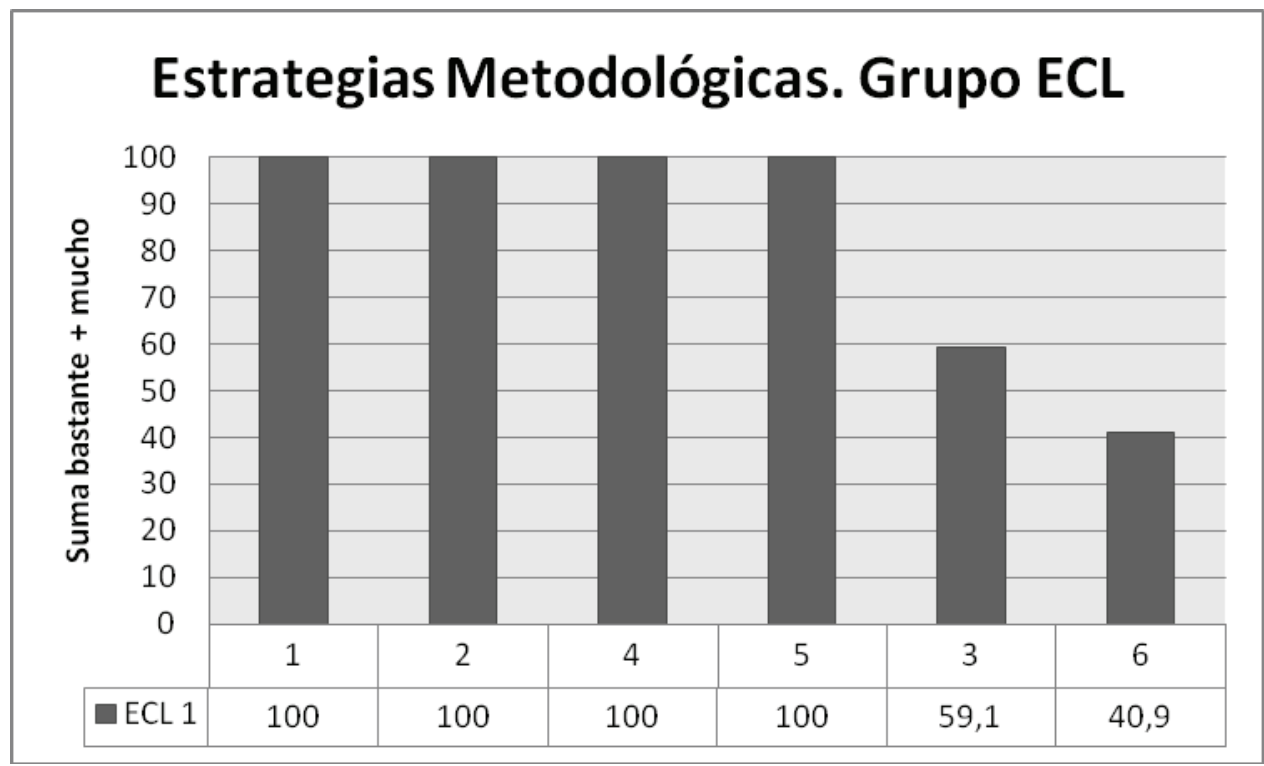

Gráfico 1: Valoración de Estrategias Metodológicas aplicadas en el grupo ECL

El cuestionario plantea una pregunta relativa a la estrategia más valiosa y efectiva que se ha aplicado en las dos semanas de curso. Como se puede comprobar, las estrategias de Aprendizaje Basado en problemas y la estrategia de aprendizaje Colaborativo son las más valoradas, especialmente el aprendizaje colaborativo. El resto de estrategias obtienen resultados muy bajos.

\begin{tabular}{|l|r|}
\hline 7.- ¿Qué estrategia te parece más valiosa y positiva? & Porcentaje \\
\hline ABP (Aprendizaje Basado en problemas) & 38,6 \\
\hline Aprendizaje colaborativo & 45,5 \\
\hline Lección Magistral & 4,5 \\
\hline Aprendizaje Auto-regulado & 6,8 \\
\hline Trabajo en grupo contractual & 4,5 \\
\hline Total & 100,0 \\
\hline
\end{tabular}

Tabla 2: valoración de estrategias metodológicas 


\section{7.-¿Qué estrategia te parece más valiosa y positiva?}

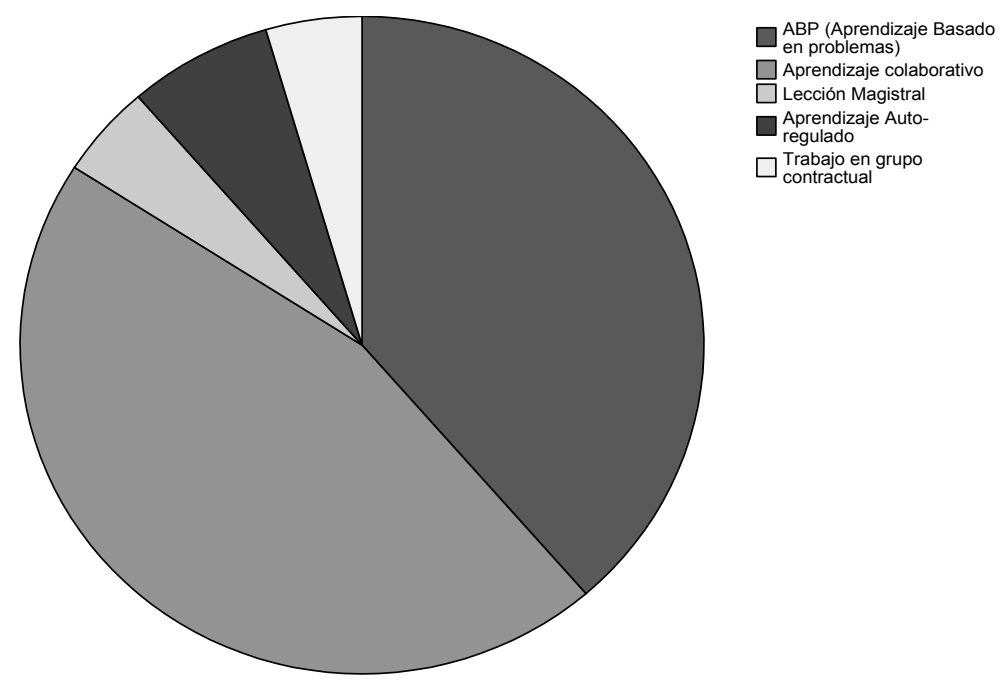

Gráfico 2: Valoración de estrategias metodológicas

El estudio cuenta con un apartado cualitativo que nos aporta datos en profundidad respecto a las opiniones y valoraciones de los participantes de la muestra a partir de instrumento que es la entrevista en profundidad. Se entrevistan a 28 sujetos en los distintos grupos.

En este caso es una entrevista semiestructurada, con la que pretendemos comprender totalmente las impresiones y experiencias de los sujetos, obteniendo información amplia y significativa. A pesar de que el uso de la entrevista requiere tiempo, puede ser complicada de analizar y el entrevistador puede influir en el entrevistado, se considera que este instrumento aporta información esencial para entender la realidad en los procesos educativos.

La complementariedad metodológica aplicada en el presente estudio aporta una triangulación de métodos. Cohen \& Marion (2000) la definen como el uso de dos o más métodos de recolección de datos en el estudio. Citan varios tipos de triangulación:

- Methodological Triangulation

- Time Triangulation (cross-sectional/longitudinal)

- Observer/Investigator Triangulation

- Theory Triangulation

- Space Triangulation

- Combined Levels of Triangulation. 
El hecho de aplicar una triangulación de métodos con un cuestionario con datos cuantitativos y unas valoraciones cualitativas reduce el sesgo del experimentador, proporciona un modelo de causalidad más amplia y compleja de la conducta, reduce errores de teorías ligadas al método y reduce errores de teorías ligadas a la cultura, lo que significa que algunas teorías se basan en la observación de una cultura.

Para analizar la información de la entrevistas rigurosamente, se analiza la información aportada y se codifica con el programa HyperResearch. Cada factor que aportan los entrevistados se codifica para un posterior análisis de las frecuencias de unos factores que se repiten y que mantienen los sujetos de la muestra.

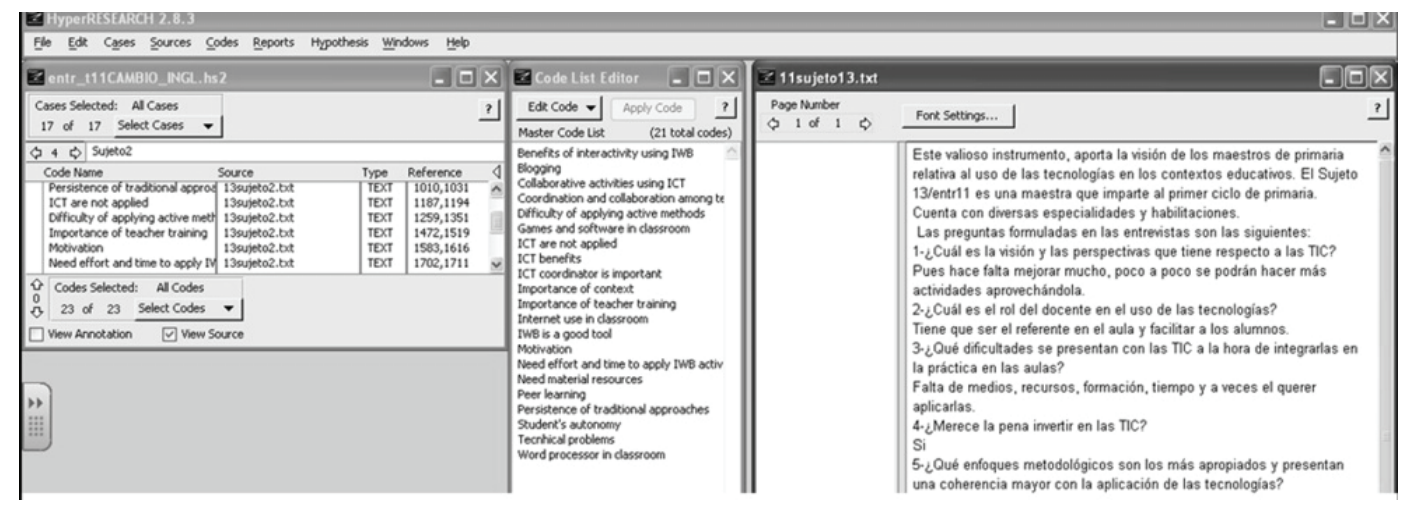

Figura 4: Uso del programa HyperResearch.

Los datos obtenidos en las entrevistas destacan una serie de factores muy destacados por los 28 sujetos entrevistados. Se aprecian valores muy altos respecto a unos enfoques activos necesarios para aprender por competencias, la importancia del ABP, la necesidad de una formación del profesorado y sobre todo la importancia del aprendizaje colaborativo, que obtiene los valores más altos. El hecho de reconocer que se mantienen enfoques tradicionales en la enseñanza, tiene valores muy altos.

Otros factores cuentan con cierto nivel de valoración por parte de la muestra, como la importancia del contexto de los alumnos, la motivación, necesidad de intencionalidad pedagógica de los docentes y recursos materiales entre los que se encuentra la $\mathrm{Pi}$ zarra Digital Interactiva (PDI) 


\begin{tabular}{|c|c|c|c|c|c|c|}
\hline \multicolumn{7}{|l|}{2 HyperRESEARCH 2.8.3 } \\
\hline \multicolumn{7}{|l|}{ File Edit Cases Sources Codes } \\
\hline \multicolumn{7}{|l|}{2 Code Frequency } \\
\hline Code & Total & Min & Max & Mean & StdDev & Bar Graph \\
\hline Aprendizaje auto regulado & 5 & 0 & 1 & 0.357 & 0.497 & \\
\hline Blogging & 2 & 0 & 1 & 0.143 & 0.363 & \\
\hline Enfoques activos para apr. por competencias & 26 & 0 & 6 & 1.857 & 1.834 & \\
\hline Falta tiempo para aplicar las TIC & 3 & 0 & 1 & 0.214 & 0.426 & \\
\hline Importancia de la leccion magistral & 2 & 0 & 2 & 0.143 & 0.535 & \\
\hline Importancia de un responsable o coordinador TIC & 3 & 0 & 1 & 0.214 & 0.426 & \\
\hline Importancia del aprendizaje colaborativo & 34 & 0 & 6 & 2.429 & 2.377 & \\
\hline Importancia del trabajo en grupo contractual & 2 & 0 & 1 & 0.143 & 0.363 & \\
\hline Importancia y efectividad del ABP & 18 & 0 & 6 & 1.286 & 1.684 & \\
\hline La importancia de la formacion de profesorado & 28 & 0 & 4 & 2 & 1.24 & \\
\hline La importancia del contexto en el que viven los alumnos & 11 & 0 & 3 & 0.786 & 0.893 & \\
\hline Motivacion & 15 & 0 & 4 & 1.071 & 1.072 & \\
\hline Necesidad de intencionalidad pedagogica de los docentes & 9 & 0 & 2 & 0.643 & 0.633 & \\
\hline Necesidad de recursos materiales & 19 & 0 & 2 & 1.357 & 0.633 & \\
\hline No se aplican las TIC & 8 & 0 & 2 & 0.571 & 0.646 & \\
\hline PDI importante & 15 & 0 & 3 & 1.071 & 1.207 & \\
\hline Presencia de enfoques tradicionales & 30 & 0 & 8 & 2.143 & 1.994 & \\
\hline Presentaciones y Power Point & 2 & 0 & 1 & 0.143 & 0.363 & \\
\hline Problemas tecnicos que dificultan en clase & 4 & 0 & 1 & 0.286 & 0.469 & \\
\hline Se utilizan las TIC con enfoque colaborativo & 6 & 0 & 2 & 0.429 & 0.852 & \\
\hline Uso de Internet en el aula & 4 & 0 & 3 & 0.286 & 0.825 & \\
\hline
\end{tabular}

Tabla 3: Frecuencias de los códigos en las entrevistas en profundidad.

\section{Conclusiones}

A partir de los datos obtenidos en el cuestionario, las entrevistas en profundidad y la práctica desarrollada en el curso se puede concluir que se obtienen valoraciones muy positivas respecto a las estrategias metodológicas relativas al Aprendizaje Colaborativo y el Aprendizaje Basado en Problemas.

Ante la pregunta ¿Es posible aplicar unos enfoques metodológicos activos, vinculados al aprendizaje por competencias integrando las Tecnologías? Las aportaciones de los sujetos no solo creen que es posible, además consideran que es necesaria e importante su aplicación. (Cuestionario, ítems 1, 2, 4, 5 y 7. Entrevistas en profundidad, 3,7 y 9).

Además se aprecia que se mantiene una coherencia y eficacia en los aprendizajes y en el desarrollo de conocimientos, habilidades y actitudes a través de prácticas activas con el uso de la Tecnología educativa, como demuestran las valoraciones muy positivas recogidos en los instrumentos de la investigación (ítem 4).

Al analizar la práctica en el aula, con una aplicación de las estrategias metodológicas ABP y Aprendizaje Colaborativo se obtienen valoraciones muy positivas respecto a las citadas estrategias, valorando la idoneidad y eficacia de las estrategias metodológicas desarrolladas. 
Comparando nuestros hallazgos con otros estudios se constata que Dochy et al (2003) aseguran que métodos de evaluación que se centran más en el reconocimiento mostraron efectos significativamente negativos favorecen el enfoque del aprendizaje tradicional. Newman et al (2003) aseguran que para aprender conocimientos es más eficaz la enseñanza tradicional y Gijbels, Dochy, Van den Bossche \& Segers (2005) revisaron 40 estudios que informaron de resultados positivos del enfoque tradicional en el aula sobre la evaluación de conocimientos conceptuales.

Desde la perspectiva del presente estudio se muestra un desacuerdo con los estudios citados, pues a partir de los datos obtenidos en diferentes instrumentos, el proceso de aprendizaje a través de estas estrategias mejora las estructuras de aplicación del conocimiento y en las habilidades y destrezas, pero también se considera una mejora en el aprendizaje de los conocimientos, pues se mejora la motivación, la atención y el compromiso del alumnado que se traduce en resultados positivos, también en la adquisición de conceptos.

El estudio tiene como valorar el uso de la Tecnología Educativa, a través de Video conferencias, material multimedia, software de accesibilidad y Blogging, hecho que se ha desarrollado en las actividades desarrolladas con las estrategias aplicadas. Las valoraciones son positivas o muy positivas. (Cuestionario, ítems 4, 5 .Entrevistas en profundidad)

A partir de la práctica pedagógica en el aula a través en los distintos grupos heterogéneos, de distintos países, se obtienen valoraciones muy positivas de las estrategias desarrolladas. Cabe destacar que existen resultados y factores muy a tener en cuenta en las entrevistas, como es la necesaria formación del profesorado y el hecho de reconocer que todavía persisten enfoques y prácticas tradicionales en el proceso de enseñanza aprendizaje. Otro dato a tener en cuenta es que un $40,9 \%$ opina que al aprender por competencias se pretenden crear ciudadanos eficientes y competitivos dejando en un segundo plano el desarrollo de los valores, afirmación muy interesante que plantea un debate de la búsqueda de una eficacia que deja como secundario la formación en valores.

En definitiva, como conclusión general se puede afirmar que los sujetos del estudio consideran que las estrategias de Aprendizaje Basado en Problemas y el Aprendizaje Colaborativo aportan unos beneficios notables en la práctica en el aula, que se ven reforzados y mejorados con la aplicación de la Tecnología Educativa. Estos enfoques son los que propician un desarrollo y un aprendizaje por competencias que demanda la exigente sociedad actual. La aportación y beneficios de las estrategias ABP y Aprendizaje Colaborativo aplicadas e integradas desde una perspectiva reformista con la Tecnología Educativa, mejoran los procesos de aprendizaje en la adquisición de conocimientos, destrezas, habilidades y actitudes a través de habilidades de pensamiento crítico y comprensivo, tomar decisiones, motivación, interactividad, autonomía, creatividad y resolución de problemas. 


\section{Referencias bibliográficas}

AREA, M (2007). Algunos principios para el desarrollo de buenas prácticas pedagógicas con las TIC en el aula. Comunicación y Pedagogía, 222, 42-47.

AVIRAM, R. (2002). ¿Conseguirá la educación domesticar a las TIC?. Ponencia presentada en el II Congreso Europeo de Tecnología de la Información, Barcelona, junio 2002.

BARAB, SASHA A. KENNETH E. HAY, MICHAEL BARNETT AND KURT SQUIRE (2001).. Constructing Virtual Worlds: Tracing the Historical Development of Learner Practices, Cognition And Instruction, 19(1), 47-94

BARROWS, H. S. (2002). Is it Truly Possible to Have Such a Thing as dPBL? Distance Education, 23(1), 119-122.

BRUFFEE, K. A. (1987). The art of collaborative learning, Change, 19 (2), $42-47$

CALVO-BERNARDINO, A.; MINGORANCE-ARNÁIZ, A. (2010). Evaluación continua de conocimientos vs de competencias: Resultados de la aplicación de dos métodos valorativos diferentes. Revista de Investigación Educativa, 28 (2), 361- 383

CANO, $\mathrm{M}^{\circ}$ E. (2008). La evaluación por competencias en la educación superior. Revista de currículum y formación del profesorado, 12 (3), 220-235

COHEN, L; MANION, L \& MORRISON, K (2000), Research Methods in Education (5th edition). London, RoutledgeFalmer.

DOCHY, FILIP, SEGERS, MIEN, VAN DEN BOSSCHE, PIET, \& GIJBELS, DAVID (2003). Effects of problema-based learning: a meta-analysis. Learning and Instruction, 13, 533-568

DULAC, J. (2006). La Pizarra Digital, ¿Una nueva metodología en el aula? Memoria final del proyecto de investigación educativa. Convocatoria MEC 2005. En http://www.dulac.es/investigaciones/pizarra/Informe\%20final.\%20Web.pdf. Consultado el 16/03/2012

GIJBELS, D., DOCHY, F., VAN DEN BOSSCHE, P., AND SEGERS, M. (2005). Effects of problem-based learning: a meta-analysis from the angle of assessment. Review of Educational Research, 75(1), 27-61

JOHNSON, R. T., \& JOHNSON, D. W. (1986). Action research: Cooperative learning in the science classroom. Science and Children, 24, 31-32

LAW, N., PELGRUM, W.J. \& PLOMP, T. (eds.) (2008). Pedagogy and ICT use in schools around the world: Findings from the IEA SITES 2006 study. Hong Kong: CERC-Springer

NEWMAN, M. (2003). Special Report 2: A pilot systematic review and meta-analysis on the efectiveness of Problem Based Learning. ITSN Learning and Teaching Support Network. Middlesex University, UK. 
RUIZ, J.M. (2009). Evaluación del diseño de una asignatura por competencias, dentro del EEES, en la carrera de Pedagogía: Estudio de un caso real. Revista de Educación. INESE. 2009. MEC. Madrid.

RUIZ, J.M. (2010). La enseñanza de competencias básicas con la pizarra interactiva en el titulo de grado a través del campo virtual. Revista Relada 4 (1): 50-61, 2010 ISSN: 1988-5822.

RUÉ, J; AMADOR, M; GENÉ, J; RAMBLA, F. X; PIVIDORU, I; TORRESHOSTENCH, O; BOSCO, A; ARMENGOL, J; FONT, A (2009). Evaluar la calidad del aprendizaje en educación superior: el modelo eca08 como base para el análisis de evidencias sobre la calidad de la e-a en E-Superior, en Red-U Revista de Educación Universitaria. 3, 1-22

RUE, J., FONT, A., CEBRIAN, G. (2011). El ABP, un enfoque estratégico para la formación en Educación Superior. Aportaciones de un análisis de la formación en Derecho. REDU-Revista de Docencia Universitaria, 9 (1), 25-44. Número Monográfico. Número especial dedicado al Aprendizaje Basado en Problemas. http://redaberta.usc.es/redu/index.php/REDU.

SÁEZ LÓPEZ, J.M. (2011). Ponencia. Estrategias Metodológicas prácticas para la Evaluación de las Competencias Profesionales. Puebla. México. Septiembre de 2011

SCAGNOLI, N.I. (2005). Estrategias para motivar el aprendizaje colaborativo en cursos a distancia. College of Education. Urbana- Champaign: University of Illinois

SHERIDAN, J. (et.al.) (1989). Collaborative Learning. Notes from the Field. College Teaching, 37(2), 49-53

STROBEL, JOHANNES AND VAN BARNEVELD, ANGELA (2008). "When is PBL More Effective? A Meta-synthesis of Meta-analyses Comparing PBL to Conventional Classrooms," Interdisciplinary Journal of Problem-based Learning : Vol. 3: Iss. 1, Article 4. Available at: http://docs.lib.purdue.edu/ijpbl/vol3/iss1/4

WAGGONER, M. (1992). A case study approach to evaluation of computer conferencing. In: Kaye, A. R. (Ed.), Collaborative learning through computer conferencing. The Najaden papers. NATO ASI Series F: Computer and Systems Sciences, (90) Berlin: Springer-Verlag. p.137-146.

YÁNIZ, C. (2008). Las competencias en el currículo universitario: implicaciones para diseñar el aprendizaje y para la formación del profesorado. Red U. Revista de Docencia Universitaria, 6 (1), 1-14. En .http://redaberta.usc.es/redu/index.php/REDU/ article/view/59/41. Consultado el 16/03/2012 


\section{Correspondencia con los autores}

José María Ruiz Ruiz

Departamento Didáctica y Organización Escolar

Facultad de Educación

Universidad Complutense de Madrid

Avda. Juan XXIII, s/n. 28040 Madrid

Tlf.: 630568745

E-mail: jmrruiz@edu.ucm.es

Jose Manuel Sáez López

Avenidad de la Mancha, $\mathrm{N}^{\circ} 6,6^{\circ} \mathrm{A}$

Universidad de Castilla la Mancha

Albacete (AB) 02006

Tlf.: 657185475

E-mail: Joshhe1977@yahoo.es 